\title{
Effect of Extrusion Strain Path on Microstructure and Properties of AZ31 Magnesium Alloy Sheet
}

\author{
Qing-Shan Yang ${ }^{1,2} \cdot$ Bin Jiang $^{2} \cdot$ Zu-Jian Yu ${ }^{1} \cdot$ Qing-Wei Dai $^{1} \cdot$ Su-Qin Luo ${ }^{2}$
}

Received: 13 April 2015/Revised: 6 August 2015/Published online: 12 October 2015

(C) The Chinese Society for Metals and Springer-Verlag Berlin Heidelberg 2015

\begin{abstract}
The mechanical properties of AZ31 magnesium alloy sheets processed by different extrusion strain paths were examined in correlation with concurrent microstructure and texture evolution. The conventional extrusion (CE) and asymmetric extrusion (ASE) paths were performed on Mg alloy sheets. The textures at near surface and mid-layer of ASE sheets were various throughout sheet thickness direction as a result of extra asymmetric shear strain. This can stimulate the orientation of (0002) basal planes to incline approximately $12^{\circ}$ toward the shear direction. Moreover, the basal texture of ASE sheet was weakened compared with CE one. Enhancing the ambient formability of extruded Mg alloy sheet fabricated by ASE path was accomplished by the tilted weak basal texture.
\end{abstract}

KEY WORDS: Mg alloy; Extrusion; Microstructure; Texture; Mechanical property; Formability

\section{Introduction}

Magnesium ( $\mathrm{Mg}$ ) alloys are one of the most attractive options for the promising applications in the automotive, aircraft and $3 \mathrm{C}$ products because of their high specific strength, low density, high electromagnetic shielding and so on [1-4]. However, Mg alloys exhibit the poor formability and the limited ductility at room temperature. This was ascribed to a limited number of active slip systems owing to their hexagonal close-packed (hcp) crystal structure [5-10]. The prevailing slip system of $\mathrm{Mg}$ alloy is the slip in the close-packed direction $<11 \overline{2} 0>$ or $<a>$ on (0002) basal plane at room temperature [11-14]. The

Available online at http://link.springer.com/journal/40195

Qing-Shan Yang

cquyqs@163.com

1 School of Metallurgy and Material Engineering, Chongqing University of Science and Technology, Chongqing 401331, China

2 Chongqing Academy of Science and Technology, Chongqing 401123, China ambient formability of $\mathrm{Mg}$ alloy sheets strongly depends on the influence of the initial crystallographic texture of (0002) basal plane. Consequently, growing interest is attracted in deforming $\mathrm{Mg}$ alloys at elevated temperatures. $\mathrm{Mg}$ alloys sheet usually gives rise to a strong basal texture during the primary process such as hot rolling and extrusion. This can result in the comparably limited room temperature formability, strong anisotropy and the compression-tension asymmetry, limiting the expanded applications of $\mathrm{Mg}$ alloy sheets [15-17]. Thus, the texture control during the primary process should be considered as an effective way to enhance the formability in the following process.

Hot and warm thermo-mechanical works are of great importance because they are not only the workability improved but also the final microstructure and properties ameliorated. A good deal of methods including equalchannel angular pressing (ECAP) [18], different speed rolling (DSR) [19], alloying [20] and heat treatment [21] has been developed to modify the basal texture of Mg alloy sheet. These methods were aimed to modify the texture of $\mathrm{Mg}$ alloy sheets, including the tilted orientation, the inclined degree of the basal plane and the texture intensity. 
However, some technologies used a great variety of repetitive processes and were unfavorable for the thin sheet fabrication. Here, a suitable process of asymmetric extrusion was performed to fabricate the AZ31 alloy sheet, which was expected to introduce the great asymmetric shear deformation during the hot extrusion process in one extrusion pass. This work was a possible texture evolution enhancing mechanical behavior of the extruded $\mathrm{Mg}$ alloy sheets. The microstructure and mechanical responses were examined and discussed.

\section{Experimental}

Cylindrical cast ingots of AZ31 Mg alloy (Mg-3.07, Al$0.78, \mathrm{Zn}-0.38 \mathrm{Mn}$, in $\mathrm{wt} \%$ ) with $82 \mathrm{~mm}$ in diameter were homogenized at $430{ }^{\circ} \mathrm{C}$ for $2 \mathrm{~h}$. The extrusion processes were conducted by conventional extrusion (CE) and asymmetric extrusion (ASE) at $430{ }^{\circ} \mathrm{C}$. The cast $\mathrm{Mg}$ alloy ingots were extruded to the sheets of $1 \mathrm{~mm}$ in thickness (ND) and $56 \mathrm{~mm}$ in width (TD) with the extrusion ratio of 101:1. The extrusion speed was $20 \mathrm{~mm} / \mathrm{s}$. Here, the ED, TD and ND denote extrusion direction, transverse direction and normal direction, respectively.

Dog-bone tensile samples of $12 \mathrm{~mm}$ in gage length, $6 \mathrm{~mm}$ in width and $1 \mathrm{~mm}$ in thickness were machined from the extruded AZ31 alloys sheets. Tensile tests were conducted on a CMT6305-300KN universal testing machine at the initial strain rate of $10^{-3} \mathrm{~s}^{-1}$ at room temperature. The uniaxial tensions are performed at the angles of $0^{\circ}, 45^{\circ}$ and $90^{\circ}$ between the tensile direction and extrusion direction, respectively.

The microstructures were examined using optical microscopy (OM) and scanning electron microscopy (SEM). The crystal orientation was examined by electron backscattered diffraction (EBSD). Following mechanical polishing, the specimens were prepared for EBSD by electropolishing at $20 \mathrm{~V}$ for $\sim 150 \mathrm{~s}$ in a AC2 solution at $-10{ }^{\circ} \mathrm{C}$. EBSD was operated at $20 \mathrm{kV}$ with a sample tilt of $70^{\circ}$. EBSD data were acquired using HKL Chanel 5 System equipped FEI Nova 400 FEG-SEM. The X-ray texture analysis was carried out by Rigaku D/Max 2500. The pole figures and EBSD maps were measured at top surface, midlayer (ND, $0.5 \mathrm{~mm}$ in thickness was removed from the surface) and bottom surface of ASE sheets, respectively.

\section{Results and Discussion}

Figure 1 shows the schematic diagram of flow passage in $\mathrm{CE}$ and ASE dies. A great deal of the asymmetric shear strain deformation was recommended through the gap between the upper and lower surfaces in the ASE die. This equipped with a different parallel flow passage length. An asymmetric shear strain region is defined by the area at the entrance of ASE die, as shown in Fig. 1b. A finite element model (FEM) was applied to examine the effective strain and the velocity of $\mathrm{Mg}$ alloy sheets during the ASE process. This was coded on the basis of the rigid-viscoplastic finite element method. The extrusion dies were treated as rigid bodies, while $\mathrm{Mg}$ alloy was treated as a rigid-viscoplastic material. All the analyses were performed under the assumption that conditions were isothermal. The FEM results of effective strain and the velocity distribution at top, middle and bottom layers of $\mathrm{Mg}$ alloy sheets are shown in Fig. 2. The FEM simulation was as a function of distance from the entrance in terms of the velocity and strain. The values of velocity and effective strain gradually increased from the entrance to the outlet of the shear zone. In addition, the velocity and strain at top surface show the maximum value. It can be seen that there exists a gradient of the velocity and strain throughout the normal direction of the extruded $\mathrm{Mg}$ alloy sheets.

Figure 3 shows optical micrographs of AZ31 alloy sheets at top surface, mid-layer and bottom surface at the shear zone in the ASE dies during the hot extrusion process. Here, the diagrammatic sketch of the sample was also shown in the figure. It can be seen that the sheets exhibit quite different microstructures in the through-thickness direction at the shear zone. Top surface microstructure has finer dynamically recrystallized (DRX) grains, which indicates that the effective strain is larger under this shear strain path. The top surface would produce the greatest maximum deviation, and the bottom surface would produce the least average on the basis of the FEM simulation results. The hot extrusion process generally includes the effects by compressive deformation and shear strain simultaneously. Reduction in area by the asymmetric shear strain occurs throughout the whole sheet thickness during the extrusion process in one ASE pass [22]. Thus, the variety in microstructure evolution of ASE sheets was generated by the deformation asymmetry.

The microstructure observations (ED-ND plane) of AZ31 alloy sheets processed by CE and ASE paths are depicted in Fig. 4. CE sheet was homogeneous and composed of equiaxed DRX grains of about $12 \mu \mathrm{m}$, as shown in Fig. 4a. On the contrary, the ASE sheet exhibited quite different microstructures throughout thickness direction (ND). The ASE sheets are behaved by inhomogeneous microstructure with finer DRX grains embedded in a large zone of elongated grains. Kim et al. [23, 24] pointed out the dynamical recrystallization was differently developed during the asymmetric rolling process, which was due to the difference of shear strain deformation in normal direction. It was of interest to see that there exists a slight grain size gradient of ASE sheets throughout the sheet 

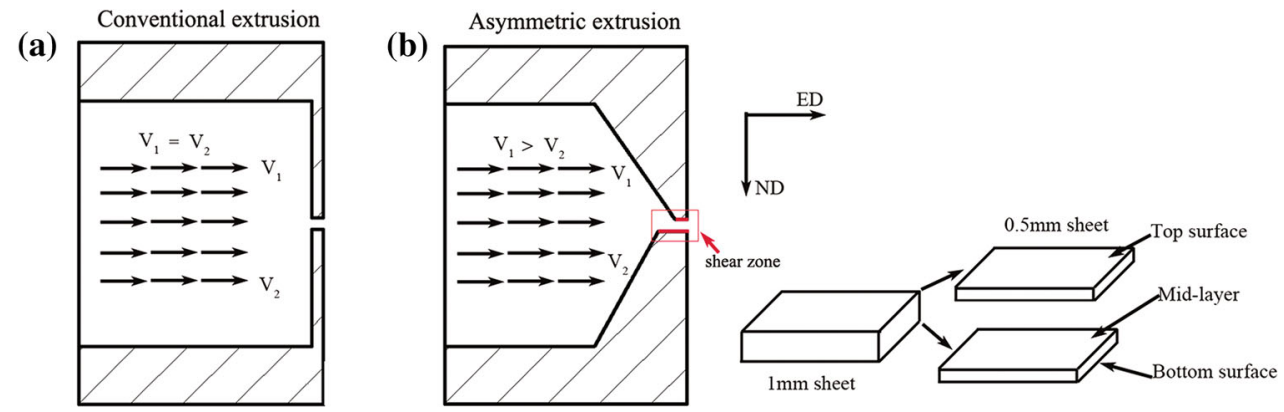

Fig. 1 Schematic diagram of the extrusion die: a conventional extrusion die; $\mathbf{b}$ asymmetric extrusion die
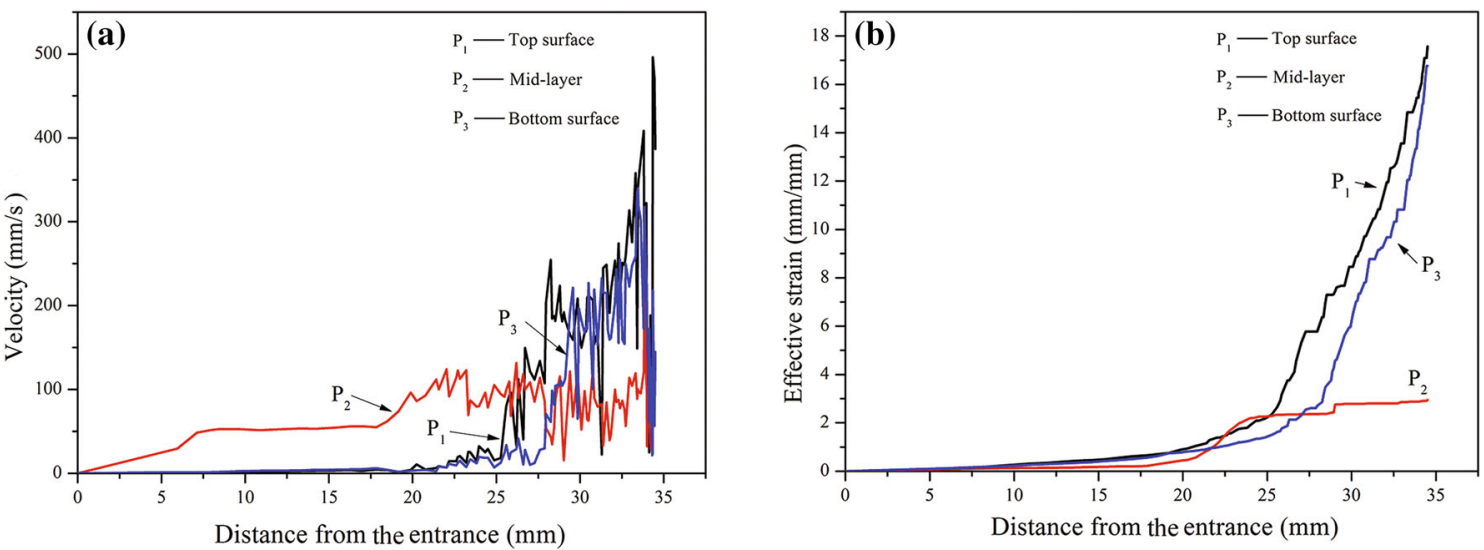

Fig. 2 FEM results of the effective strain $\mathbf{a}$, the velocity $\mathbf{b}$ distribution of $\mathrm{Mg}$ alloy sheet during the ASE process

thickness direction. The relationship between the average grain size $(d)$ and the Zener-Hollomon parameter $(Z)$ can be given by $\ln d=A+B \ln Z$ during the dynamic recrystallization. Here, the temperature-corrected strain rate $Z$ can be given by $Z=\varepsilon \cdot \exp (Q / R T)$, where $\varepsilon, Q, T$ and $R$, respectively, are the strain rate, the activation energy for the deformation, the temperature and the gas constant [25]. It is roughly reached the decision that more strain rate can generate the smaller grain size according to the above equations based on the FEM simulation results. It can be seen that the effective strain or the velocity is uneven during the ASE process from the simulation results. This indicates that the degree of DRX throughout thickness direction of sheets is different owing to the asymmetric shear strain. Thus, the position of the sheets where the velocity or effective strain was larger had finer DRX grains under this strain path.

To examine the microstructures in detail, EBSD orientation maps of the CE and ASE sheets (at top surface, midlayer and bottom surface) are displayed in Fig. 5. (0002) pole figures of AZ31 alloy sheets are shown in Fig. 6. It can be seen that CE sheets processed the remarkably basal texture and homogeneous microstructure after the hot extrusion. That is, $\langle 11 \overline{2} 0>$ direction in most grains orientated parallel to the extrusion direction. This strong basal texture results in a poor deformation capability and a stronger anisotropy, which finally brings about a poor ductility at room temperature [26]. As shown in Fig. 6b, the (0002) basal texture of the top region of the ASE sheets weakens and tilts about $12^{\circ}$ toward ED compared with CE ones. The coarse grains of ASE sheets have been elongated and rotated away from the basal texture due to the asymmetric shear. On the contrast, the fine grains have been fully dynamically recrystallized toward basal texture orientation. Since the effective strain at mid-layer of sheet was uniform, the microstructure showed the typical basal texture and consisted of coarse grains. The asymmetric extrusion can impose the maximum asymmetric shear strain deformation, which can make the basal texture rotate toward the imposed shear direction in the successive pass during hot extrusion process [27-29]. ASE path is more efficient in generating the plastic deformation because it developed the additional shear strain for a given reduction in thickness compared with CE one [30, 31]. The tilted weak texture of ASE sheets was achieved as a result of the characteristic of the asymmetric extrusion process. Thus, the basal texture of top and bottom surfaces were both inclined. It can be seen that there exists a slight decrease 

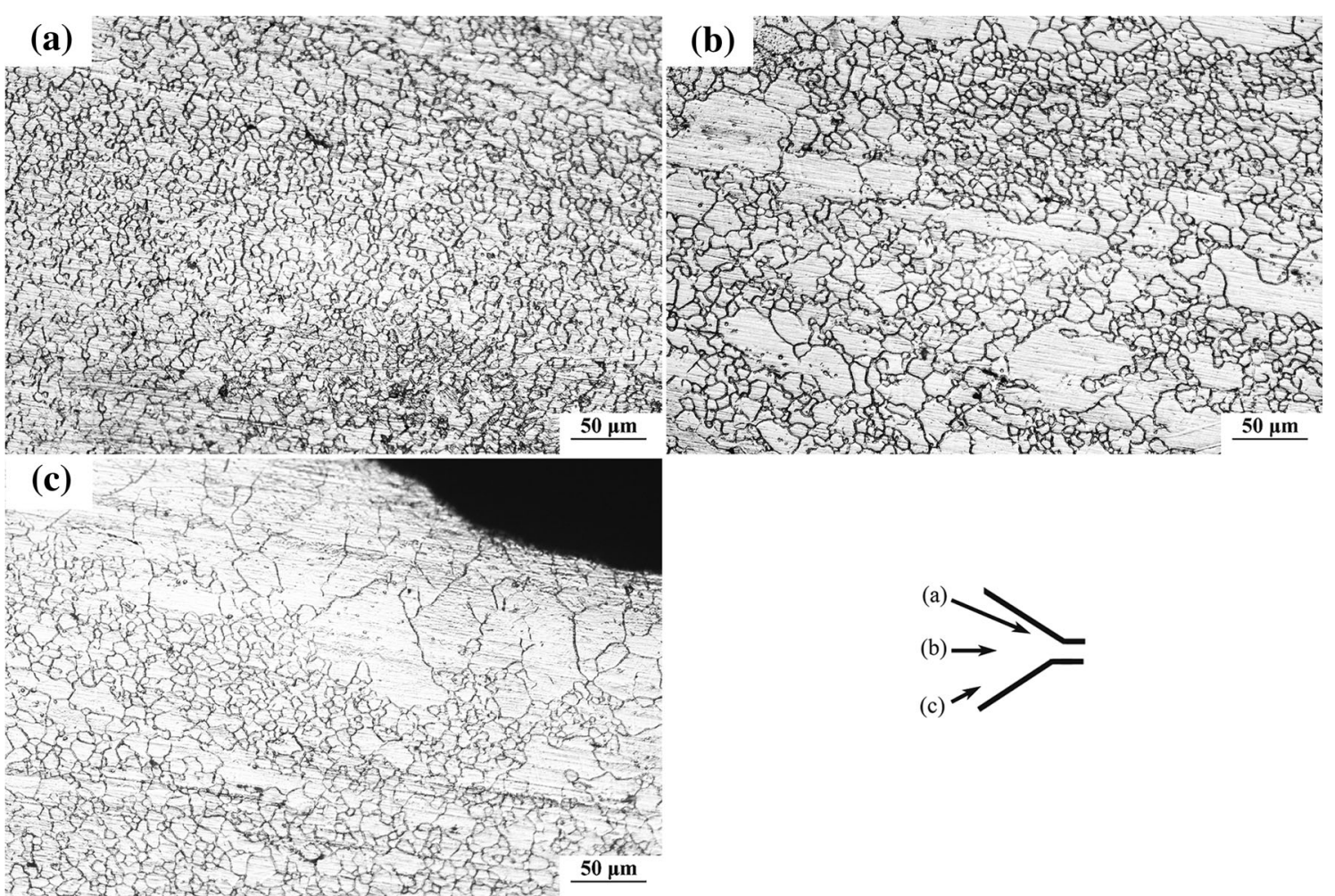

Fig. 3 Optical micrographs of AZ31 alloy at the shear zone: a top surface, b mid-layer, $\mathbf{c}$ bottom surface in the ASE dies during the hot extrusion process
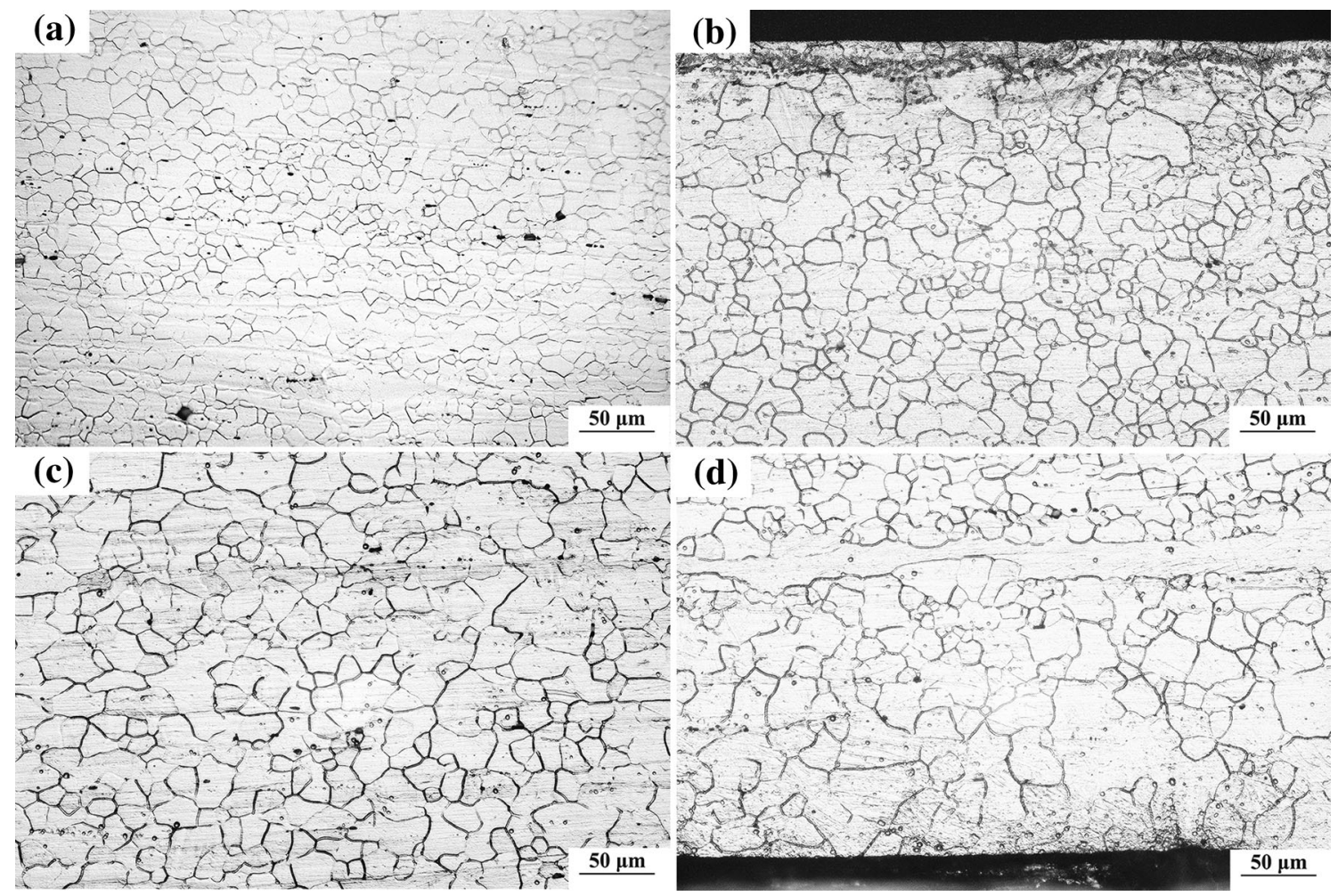

Fig. 4 Optical micrographs of the CE sheet $\mathbf{a}$, the ASE sheet at top surface $\mathbf{b}$, mid-layer $\mathbf{c}$, bottom surface $\mathbf{d}$ 
CE-sample
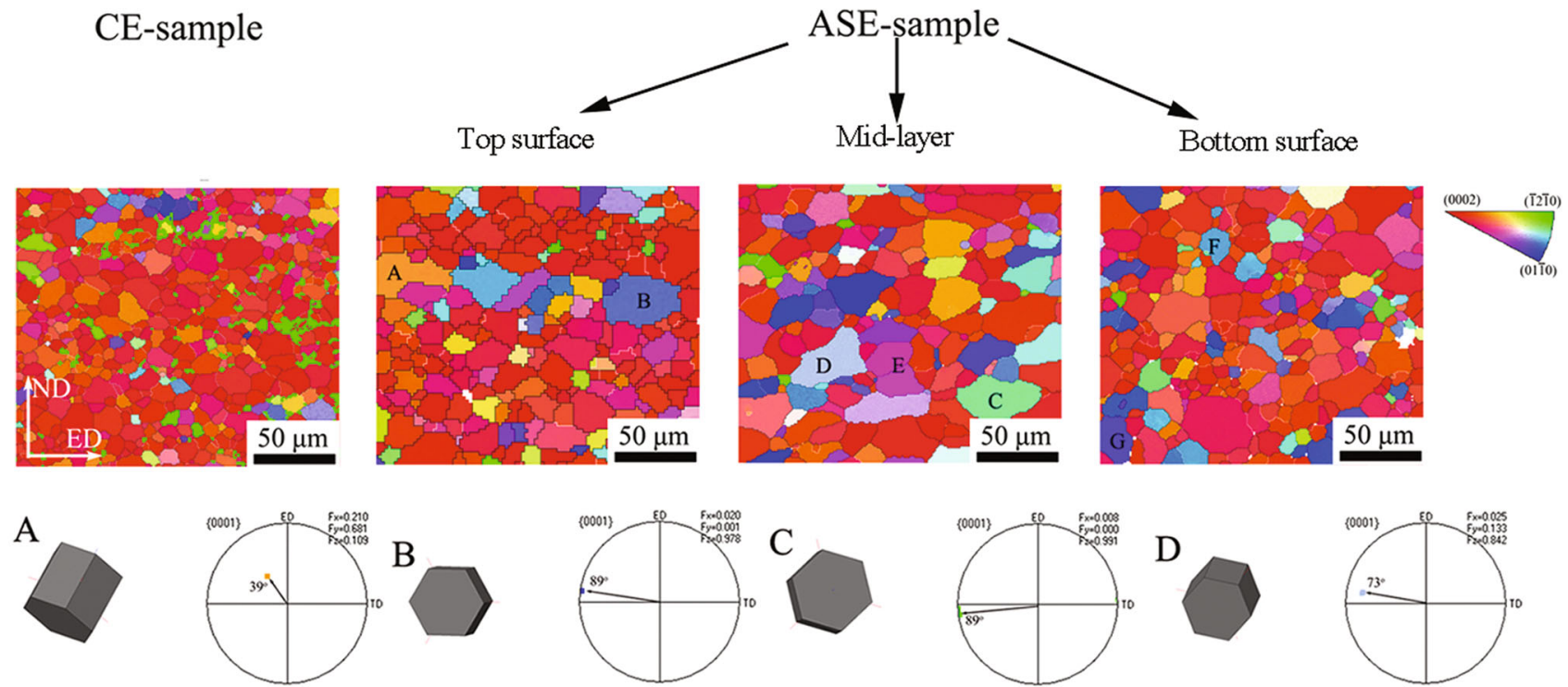

$\mathrm{D}$
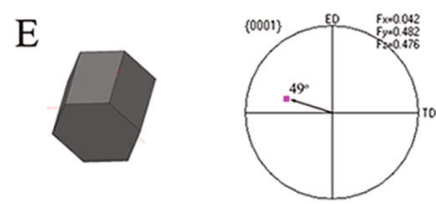

$\mathrm{F}$
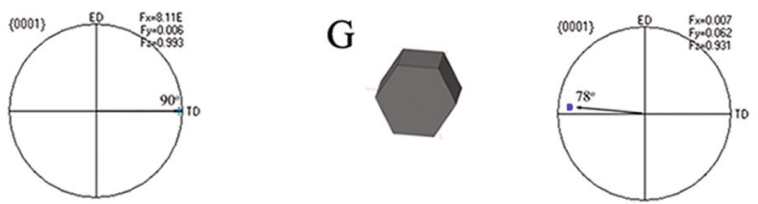

Fig. 5 EBSD orientation maps of CE sheets, ASE sheets at top surface, mid-layer and bottom surface. Here, points $a, b, c, d, e, f, g$ denote the $c$ axis correspond to the grains, respectively

(a)

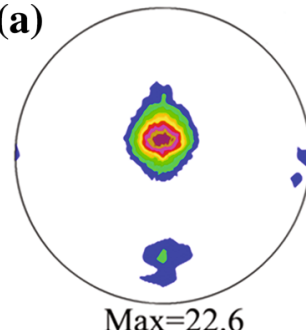

(c)

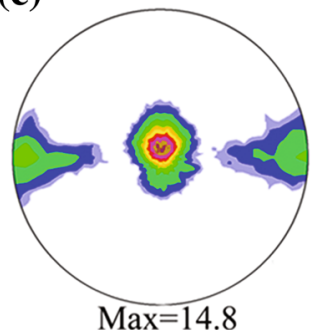

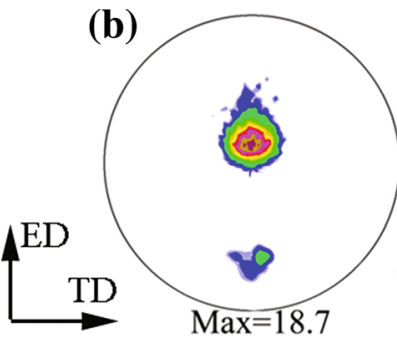

(d)

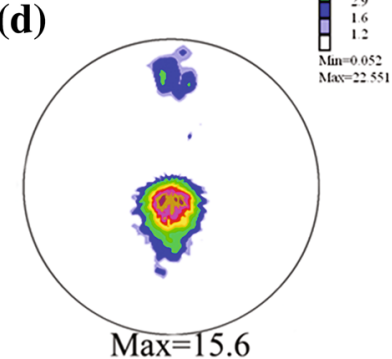

Fig. 6 (0002) pole figures of AZ31 alloy sheets: a CE sheet; the ASE sheet at top surface $\mathbf{b}$, mid-layer $\mathbf{c}$, bottom surface $\mathbf{d}$

gradient for the basal texture of ASE samples throughout the sheet thickness direction. The $c$-axis of the grains $\mathrm{A}, \mathrm{B}$, C, D, E, F and G tilted about $39^{\circ}, 89^{\circ}, 89^{\circ}, 73^{\circ}, 49^{\circ}, 90^{\circ}$ and $78^{\circ}$ away from the basal plane, respectively, as seen from Fig. 5. The majority of the grains were more suitable for prismatic $<a>$ slip than basal $<a>$ slip due to the

shear strain deformation [32, 33]. The prismatic $<a>$ slip was the main mode of the strain accommodation. Thus, the activation of prismatic $\langle a\rangle$ slip results in a rotation of the basal plane from the ED toward the imposed shear direction.

The true stress-strain curves measured at different directions tilting $0^{\circ}, 45^{\circ}$ and $90^{\circ}$ to ED for CE and ASE sheets at room temperature are expressed in Fig. 7. The curves of CE sheet confirm the strong anisotropy of stress-

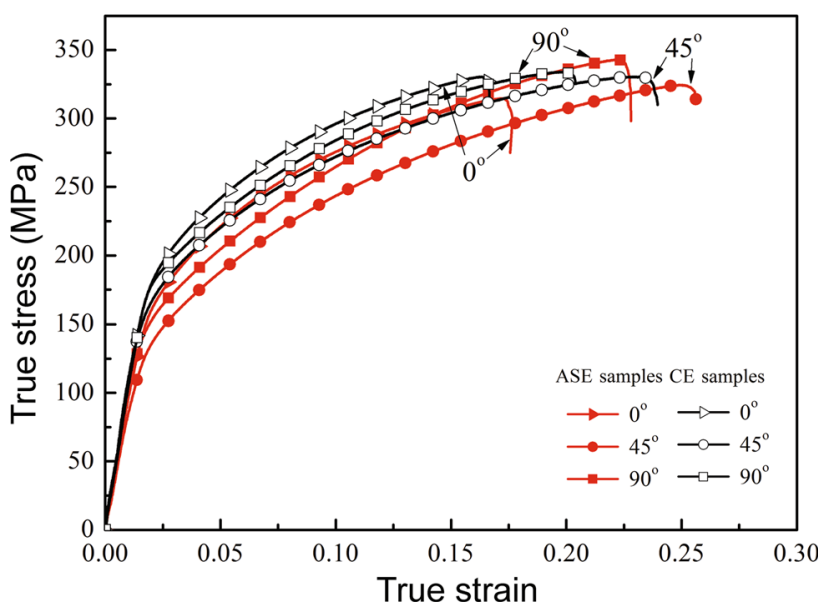

Fig. 7 True stress-strain curves during tensile tests at different directions of CE and ASE sheets 
Table 1 Results of the tensile tests carried out at the tensile directions of $0^{\circ}, 45^{\circ}$ and $90^{\circ}$

\begin{tabular}{|c|c|c|c|c|c|c|c|c|c|c|c|c|c|c|}
\hline \multirow[t]{2}{*}{ Sample } & \multicolumn{3}{|c|}{ UTS (MPa) } & \multicolumn{3}{|c|}{ YS (MPa) } & \multicolumn{3}{|c|}{$E_{\mathrm{u}}(\%)$} & \multicolumn{5}{|c|}{ Average value } \\
\hline & $0^{\circ}$ & $45^{\circ}$ & $90^{\circ}$ & $0^{\circ}$ & $45^{\circ}$ & $90^{\circ}$ & $0^{\circ}$ & $45^{\circ}$ & $90^{\circ}$ & UTS & YS & $E_{\mathrm{u}}$ & $n$ & $r$ \\
\hline $\mathrm{CE}$ & 332 & 323 & 331 & 161 & 148 & 167 & 15.4 & 22.1 & 19.0 & 327 & 156 & 19.7 & 0.29 & 1.64 \\
\hline ASE & 315 & 326 & 344 & 150 & 125 & 136 & 16.4 & 23.7 & 22.1 & 328 & 134 & 21.5 & 0.34 & 1.00 \\
\hline
\end{tabular}
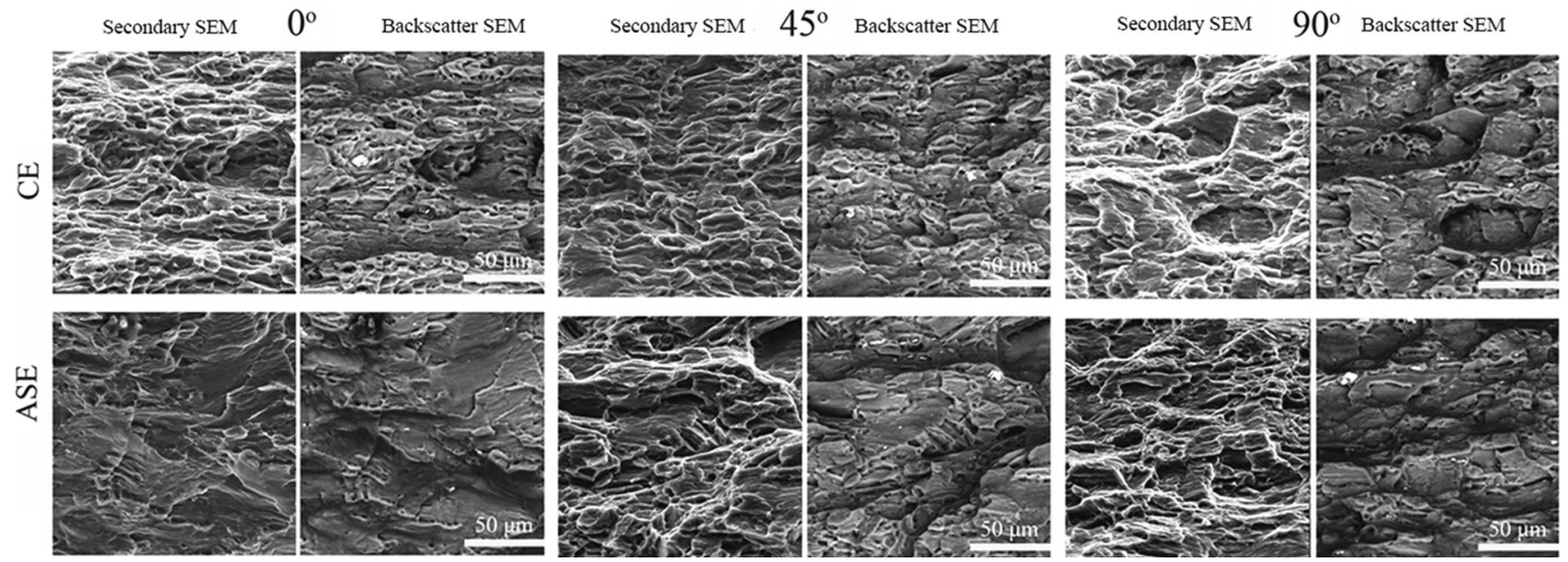

Fig. 8 Secondary and the backscatter SEM fracture images of CE and ASE specimens

strain behavior with respect to the initial strong (0002) basal texture. In this case, the high stress is needed to activate the basal slips, which lead to a strong hardening behavior at the initial stage of tensile test. Most of the grains have the ideal orientation for the slip on the basal planes of the extruded $\mathrm{Mg}$ sheets. The measured macroscopic true stress $(\sigma)$ and true plastic strain $(\varepsilon)$ can be related to the resolved shear stress $(\tau)$ and the shear strain $(\gamma)$ on basal planes. The relations can be given by $\varepsilon=m_{\mathrm{S}} \gamma$ and $\sigma=\tau / m_{\mathrm{S}}$, where $m_{\mathrm{S}}$ is the Schmid factor for the basal slip [34]. The $45^{\circ}$ sample shows a relatively low yield stress compared with those of $0^{\circ}$ and $90^{\circ}$ samples. In addition, the $45^{\circ}$ sample has the highest uniform elongation. It is expected that the Schmid factor $m_{\mathrm{S}}=\cos \lambda \cos \varphi$. Here, $\lambda$ is the angle between the stress axis and the slip direction, and $\varphi$ is the angle between the stress axis and the slip plane normal, respectively. When $m_{\mathrm{S}}=0.5\left(\lambda=45^{\circ}\right.$, $\left.\varphi=45^{\circ}\right)$, it was near the maximum [35].

The mechanical properties of the yield strength (YS, $0.2 \%$ offset strength), the ultimate tensile strength (UTS), the uniform elongation $\left(E_{\mathrm{u}}\right)$, the Lankford value ( $r$ value) and the strain-hardening exponent value ( $n$ value) for the AZ31 alloy sheets are summarized in Table 1. The average value of $E_{\mathrm{u}}$ increases from 19.7 to $21.5 \%$, while YS decreases from 156 to $134 \mathrm{MPa}$. It was implied that the ductility of ASE sheet is higher than that of CE one. Meanwhile, it also illustrates that the grain size is not a dominant factor for the yield stress. The low value of the yield stress in ASE sheets is due to the difference of the basal texture. It supposes that the elongated grains of ASE sheets have different orientations with respect to the fine grains. Some small divergence of the texture was caused by the inhomogeneity of the microstructure and grain orientation because of the shear strain. Furthermore, the $n$ value increases from 0.29 to 0.34 and the $r$ value decreases from 1.64 to 1.00 simultaneously, which is attributed to the tilted weak texture. The $\mathrm{CE}$ sheets with the strong basal texture are unfavorable for the basal slip during the plastic deformation. However, a weak basal texture and inclination of the $c$-axes can favor the action of the basal slip for ASE sheets. This results in the superior stretch formability with larger $n$ value and smaller $r$ value for ASE sheets. It is well known that the shear deformation can improve the $r$ value of the sheets, and thus shear texture has often been favored over texturing by the plane strain for $\mathrm{Mg}$ alloy sheet forming [36]. The effective shear strain of ASE sheets was different throughout the sheet thickness direction. A high extra shear strain can activate the non-basal slip systems of $\mathrm{Mg}$ alloy sheets. The intense interactions of multiple deformation models would result in the rotation of the basal texture [37].

Figure 8 depicts the SEM images of CE and ASE sheets obtained by both secondary and backscattered electrons. A mass of plastic dimples and cleavage planes occur on the 
fracture of the sheets. In addition, some dimples are inside the cleavage planes, which belong to the ductile failure $[38,39]$. On the fracture surface of the CE sheet, dimples are less and shallower, while those of ASE sheets show cruder. This distinctly evinces the shear deformation at the shear zone resulting in the good plasticity during the ASE path. An additional shear at grain boundary can activate the non-basal slip systems. The intense interactions of multiple deformation models bring about the rotation of basal texture and a large uniform elongation in the sheets processed by the asymmetric extrusion. Further investigation of the microstructure is needed to make clear the deformation mechanism of the present extruded AZ31 Mg alloy sheets.

\section{Conclusions}

The present work has demonstrated that the asymmetric extrusion process can effectively modify the room temperature ductility of $\mathrm{Mg}$ alloys by altering the basal texture. The mechanical properties of AZ31 Mg alloy sheets processed by ASE path were remarkably improved compared with CE ones. Different degrees of the dynamic recrystallization were motivated by the extra asymmetric shear strain in sheet thickness direction during the ASE path, which makes the basal planes rotate toward about $12^{\circ}$ the imposed shear direction. The excellent ambient formability can be attributed to the tilted weaken basal texture, resulted in a lower YS, a larger $E_{\mathrm{u}}$, a larger $n$ value and smaller $r$ value. This approach is an efficient alternative to improve the formability of the extruded $\mathrm{Mg}$ alloys.

Acknowledgments This work was financially supported by the Postdoctoral Science Foundation of China (Nos. 2015M572451 and 2015M572447), Chongqing Science and Technology Commission (Nos. cstc2014fazktjcsf0112, cstc2014jcyjA50019 and cstc2014fazktjcsf50004), National Natural Science Foundation of China (No. 51501025), Foundation of Chongqing Municipal Education Committee (No. KJ1401321) and the Research Foundation of Chongqing University of Science and Technology (Nos. CK2013B13 and CK2014Z21).

\section{References}

[1] X.-H. Chen, L.-Z. Liu, J. Liu, F.-S. Pan, Acta Metall. Sin. (Engl. Lett.) 28, 492 (2015)

[2] A.A. Luo, J. Magn. Alloys 1, 2 (2013)

[3] D. Sorgente, L.D. Scintilla, G. Palumbo, L. Tricarico, Int. J. Mater. Form. 3, 13 (2009)

[4] R.L. Doiphode, S.V.S. NarayanaMurty, N. Prabhu, B.P. Kashyap, J. Magn. Alloys 1, 169 (2013)

[5] N. Stanford, J. Geng, Y.B. Chun, C.H.J. Davies, J.F. Nie, M.R. Barnett, Acta Mater. 60, 218 (2012)
[6] A. Hadadzadeh, M.A. Wells, J. Magn. Alloys 1, 101 (2013)

[7] M. Nebebe, J. Bohlen, D. Steglich, D. Letzig, Int. J. Mater. Form. 2, 53 (2009)

[8] Q.S. Yang, B. Jiang, G.Y. Zhou, J.J. He, F.S. Pan, Mater. Sci. Technol. 30, 227 (2014)

[9] H. Zhang, W. Jin, J. Fan, W. Cheng, H. Jørgen Roven, B. Xu, H. Dong, Mater. Lett. 135, 31 (2014)

[10] H. Zhang, G. Huang, J. Fan, H. Jørgen Roven, F. Pan, B. Xu, Mater. Sci. Eng. A 608, 234 (2014)

[11] D.L. Atwell, M.R. Barnett, W.B. Hutchinson, Mater. Sci. Eng. A 549, 1 (2012)

[12] H. Pan, F. Pan, J. Peng, J. Gou, A. Tang, L. Wu, H. Dong, J. Alloy. Compd. 578, 493 (2013)

[13] D.-H. Hou, S.-M. Liang, R.-S. Chen, C. Dong, E.-H. Han, Acta Metall. Sin. (Engl. Lett.) 28, 115 (2014)

[14] S. Liu, G. Yang, W. Jie, Acta Metall. Sin. (Engl. Lett.) 27, 1134 (2014)

[15] R. Li, F. Pan, B. Jiang, Q. Yang, A. Tang, Mater. Des. 46, 922 (2013)

[16] A.S. Khan, A. Pandey, T. Gnäupel-Herold, R.K. Mishra, Int. J. Plast. 27, 688 (2011)

[17] L.W. Meyer, M. Hockauf, B. Zillmann, I. Schneider, Int. J. Mater. Form. 2, 61 (2009)

[18] S.R. Agnew, J.A. Horton, T.M. Lillo, D.W. Brown, Scr. Mater. 50, 377 (2004)

[19] J.-H. Cho, H.-W. Kim, S.-B. Kang, T.-S. Han, Acta Mater. 59, $5638(2011)$

[20] L. Shang, S. Yue, R. Verma, P. Krajewski, C. Galvani, E. Essadiqi, Mater. Sci. Eng. A 528, 3761 (2011)

[21] W.X. Wu, L. Jin, Z.Y. Zhang, W.J. Ding, J. Dong, J. Alloys Compd. 585, 111 (2014)

[22] L.L. Chang, S.B. Kang, J.H. Cho, Mater. Des. 44, 144 (2013)

[23] W.J. Kim, S.I. Hong, J.M. Lee, S.H. Kim, Mater. Sci. Eng. A 559, 325 (2013)

[24] W.J. Kim, Y.G. Lee, M.J. Lee, J.Y. Wang, Y.B. Park, Scr. Mater. 65, 1105 (2011)

[25] L.L. Chang, Y.N. Wang, X. Zhao, J.C. Huang, Mater. Sci. Eng. A 496, 512 (2008)

[26] B. Song, R. Xin, G. Chen, X. Zhang, Q. Liu, Scr. Mater. 66, 1061 (2012)

[27] Y. Wang, S.B. Kang, J. Cho, J. Alloys Compd. 509, 704 (2011)

[28] H. Zhang, Y. Liu, J. Fan, H.J. Roven, W. Cheng, B. Xu, H. Dong, J. Alloys Compd. 615, 687 (2014)

[29] H. Zhang, G. Huang, J. Fan, H.J. Roven, B. Xu, H. Dong, J. Alloys Compd. 615, 302 (2014)

[30] Y.H. Ji, J.J. Park, Mater. Sci. Eng. A 499, 14 (2009)

[31] X. Huang, K. Suzuki, Y. Chino, M. Mabuchi, J. Alloys Compd. 537, 80 (2012)

[32] O. Sabokpa, A. Zarei-Hanzaki, H.R. Abedi, Mater. Sci. Eng. A 550, 31 (2012)

[33] D.G. Kim, K.M. Lee, J.S. Lee, Y.O. Yoon, H.T. Son, Mater. Lett. 75, 122 (2012)

[34] J.A. del Valle, F. Carreño, O.A. Ruano, Acta Mater. 54, 4247 (2006)

[35] R. Li, F. Pan, B. Jiang, H. Dong, Q. Yang, Mater. Sci. Eng. A 562, 33 (2013)

[36] X. Huang, K. Suzuki, Y. Chino, J. Alloys Compd. 509, 4854 (2011)

[37] B. Wang, R. Xin, G. Huang, Q. Liu, Mater. Sci. Eng. A 534, 588 (2012)

[38] K. Piao, J.K. Lee, J.H. Kim, H.Y. Kim, K. Chung, F. Barlat, R.H. Wagoner, Int. J. Plast 38, 27 (2012)

[39] L. Lu, T. Liu, Y. Chen, Z. Wang, Mater. Charact. 67, 93 (2012) 